\title{
Stochastic processes, galactic star formation, and chemical evolution
}

\section{Effects of accretion, stripping, and collisions in multiphase multi-zone models}

\author{
G. Valle ${ }^{1}$, S. N. Shore ${ }^{1,2}$, and D. Galli ${ }^{3}$ \\ 1 Dipartimento di Fisica "Enrico Fermi”, Università di Pisa, largo Pontecorvo 3, Pisa 56127, Italy \\ e-mail: valle@df.unipi.it \\ 2 INFN/Pisa, largo Pontecorvo 3, Pisa 56127, Italy \\ 3 INAF - Osservatorio Astrofisico di Arcetri, largo Enrico Fermi 5, Firenze 50125, Italy
}

Received 3 November 2004 / Accepted 3 February 2005

\begin{abstract}
This paper reports simulations allowing for stochastic accretion and mass loss within closed and open systems modeled using a previously developed multi-population, multi-zone (halo, thick disk, thin disk) treatment. The star formation rate is computed as a function of time directly from the model equations and all chemical evolution is followed without instantaneous recycling. Several types of simulations are presented here: (1) a closed system with bursty mass loss from the halo to the thick disk, and from the thick to the thin disk, in separate events to the thin disk; (2) open systems with random environmental (extragalactic) accretion, e.g. by infall of high velocity clouds directly to the thin disk; (3) schematic open system single and multiple collision events and intracluster stripping. For the open models, the mass of the Galaxy has been explicitly tracked with time. We present the evolution of the star formation rate, metallicity histories, and concentrate on the light elements. We find a wide range of possible outcomes, including an explanation for variations in the Galactic $\mathrm{D} / \mathrm{H}$ ratio, and highlight the problems for uniquely reconstructing star forming histories from contemporary abundance measurements.
\end{abstract}

Key words. galaxies: evolution - galaxies: abundances - galaxies: interactions

\section{Introduction}

The determination of galactic evolution over cosmic time requires the interpretation of the elemental abundance distribution as a record of changing star formation patterns. Unraveling the web of physical processes has proven a challenge, complicated by the many fundamental uncertainties associated with specific scenarios for the physical processes. A number of approaches, often complementary, have been tried. These split broadly into two categories. One approach, Chemodynamics include hydrodynamics (parameterized chemical evolution with either multiple fluids or SPH, etc.) along with the appropriate nuclear yields but generally use a simple formulation for the star formation rate as a function of gas density (see Hensler 2003 and references therein). The other uses analogs of chemical networks and either assume a time history for the star formation rate in advance (e.g. Tinsley 1980; Hellsten \& Sommer-Larsen 1992; Pagel 1998; Matteucci 2003) or adopt a nonlinear dynamical systems approach with feedback (e.g. Lynden-Bell 1975; Shore \& Ferrini 1995; Shore \& Franco 2000 ). Each has its successes in modeling some aspect(s) of the system but most share the feature that they are closed and deterministic, conditions known to be respected more as exceptions than as a rule (e.g. van Gorkem 2004). Only a few stochastic simulations have been attempted date, especially percolation models (e.g. Gerola \& Seiden 1978; Matteucci \& Chiosi 1983; Schulman \& Seiden 1986; Comins \& Shore 1990; Perdang \& Lejeune 1996) and fluctuating accretion (Copi et al. 1995; Copi 1997).

It is beyond the scope of this paper to review the assumptions of these individual approaches. Instead, we will present results of ongoing simulations, using our previously developed multi-population framework (cf. Ferrini et al. 1992; Shore \& Ferrini 1995; Shore \& Galli 2003; Valle et al. 2002), that include stochastic accretion within closed and open systems (cf. Comins \& Shore 1990). Our purpose is to show that even these relatively schematic models, which have been recently used to study the evolution of the light elements, display behavior that can inform more detailed modeling using any of the cited approaches of the dynamical and nucleosynthetic processes associated with galactic evolution.

\section{Baseline Galactic chemical evolution model: The standard model}

We begin with a description of our baseline, or standard model that has been developed for the Galaxy in a previous series of 
papers so the changes we introduce here will be understood in their proper context. The model treats evolution within a region formed by the intersection with the Galactic disk of a cylinder of radius $\sim 1 \mathrm{kpc}$ and with its axis perpendicular to the disk. The vertical height corresponds to the scale length for the halo. The cylinder is centered at the Solar galactocentric distance. By the term galaxy we mean only the actively star forming phase; a dark matter halo, constituting the bulk of the mass, is assumed to be inertly present to insure that there is no need to include dynamics explicitly in the code. Our technique uses a multi-zone, multiphase approach. Three "zones" (halo, thick disk, and thin disk) of the galaxy are initialized with only the halo, for which only the diffuse gas phase is initially included and for which primordial abundances have been assumed. The other two form by accretion. The time history of star formation is computed directly from the model equations rather than being assumed a priori without instantaneous recycling and the yields and deterministic rates are the same as used in our previous work (most recently, Valle et al. 2002). We distinguish the halo (HA), thick disk (TD) and thin disk (DI) and within each include three phases (plus an inert sink): diffuse gas, clouds, stars and remnants. All are expressed as the phase mass fraction relative to the total mass of the system. We assume that all the matter is initially in the form of diffuse gas in halo, which then begins to form the thick disk while forming clouds and then stars. Gas that falls onto the thick disk is already enriched from nucleosynthesis by halo stars. It forms clouds (through gravitational instabilities, although dynamical processes are not included explicitly in this modeling scheme). Star formation occurs both spontaneously, when stars are formed directly from the cloud phase, and by stimulated processes through the interaction of clouds and massive stars. The same processes occur in the thin disk.

The interactions among the different phases produce the time dependence of the total mass fraction in each phase and the chemical abundances in the interstellar medium (ISM) and in stars. The restitution of matter to the ISM is described by two time dependent terms: the death rate $D(t)$ (the fraction of the total mass that leaves the star phase at the time $t$ ) and the restitution rate $W(t)$ (the fraction of the mass injected in the interstellar gas from stars dying at $t$ ). The computation of these terms is based on the assumptions made for stellar evolution and nucleosynthesis (see Ferrini et al. 1992). The star formation rate (SFR) is determined consistently by the interaction among the different phases of matter and no formal time dependence for the SFR is assumed a priori. The initial mass function (IMF) adopted in this model is based on the analysis of the fragmentation of molecular clouds in the solar neighborhood (Ferrini et al. 1991), and is constant in space and time although we have tested the sensitivity of the final abundances to this IMF by comparison of the mass averaged yields using Kroupa 2002. The principal effect of the mass distribution on the final abundances is for elements whose formation has been delayed by stellar evolution with respect to the stellar birth time, such as $\mathrm{Fe}$, since no instantaneous recycling approximation (IRA) is assumed in our calculations. The results, which will be reported in a future paper, yielded no qualitative changes and thus, for correspondence with our previously published work, we maintain the original IMF here.

Matter exchanges between different zones and phases are codified by coefficients given by the typical timescale and efficiency of the related process. In our standard framework we keep these coefficients at constant values during the evolution of the system, so any time dependence arises from the variation of the mass fraction of the different phases. Some variations of the microphysics properties of the system over time might occur, which would affect the rates (Shore \& Ferrini 1995). The main rates that drive the interactions among the different phases are the spontaneous formation, the cloud-cloud collisions terms, and the star-cloud interaction terms. For a detailed description of the standard version of the model - in particular the adopted nucleosynthesis prescriptions, SNI and SNII progenitors, initial mass function - we refer the reader to Ferrini et al. (1992), Pardi \& Ferrini (1994), Ferrini et al. (1994), and Valle et al. (2002). For comparison with subsequent simulations, we show the results of the standard model in Fig. 1. The coupling between zones is obtained through the infall parameters $f_{\mathrm{H}}$ and $f_{\mathrm{TD}}$. The thick disk accumulates matter falling from the halo and in turn feeds the thin-disk zone. These two coefficients are independent and kept constant in time; they are the only truly free parameters of the model. The timescale for the formation of the thick disk from the halo is assumed to be shorter than the accretion time for formation for the thin disk during which it accretes matter from the thick disk $\left(f_{\mathrm{H}}=0.1\right.$, $f_{\mathrm{TD}}=6.5 \times 10^{-3}$ ).

The model output includes the time dependent abundances of $\mathrm{H},{ }^{2} \mathrm{D},{ }^{3} \mathrm{He},{ }^{4} \mathrm{He},{ }^{12} \mathrm{C},{ }^{13} \mathrm{C},{ }^{14} \mathrm{~N},{ }^{16} \mathrm{O},{ }^{20} \mathrm{Ne},{ }^{24} \mathrm{Mg},{ }^{28} \mathrm{Si}$, ${ }^{32} \mathrm{~S},{ }^{40} \mathrm{Ca},{ }^{56} \mathrm{Fe}$ and $s$ - and $r$-process elements (Travaglio et al. 1999). The reactions for light isotopes $(\mathrm{Li}, \mathrm{Be}, \mathrm{B})$ production have recently been inserted (Valle et al. 2002), including the Galactic cosmic ray spallation on interstellar CNO nuclei (direct processes), spallation by $\mathrm{CNO}$ cosmic ray nuclei impinging on interstellar $p$ and ${ }^{4} \mathrm{He}$ (inverse processes), and $\alpha-\alpha$ fusion reactions. The model produces good agreement with the linear trend of $\mathrm{Be}$ and $\mathrm{B}$ observed at low metallicities without other modification to the model parameters or equations.

This standard model is closed in the sense that neither accretion into, nor outflow from, the system occur. Any gas shed from the halo and thick disk is assumed to accrete ultimately onto the thin disk and the mass of the galaxy stays constant. The evolution computed using constant rate coefficients and constant IMF in time. No instantaneous recycling is used, the metallicity evolution is explicitly computed for a fixed initial mass function by including stellar mass-dependent nucleosynthetic yields. Turbulent mixing and advective transport are not included so the treatment is effectively radially local and vertically stratified, in contrast to chemodynamical models, although it should be complementary (for a comprehensive survey of the various modeling schemes, see Hensler et al. 2003). In the last panel of Fig. 1, and in subsequent figures, we use the SFR at any time to represent the number of stars formed at that instant with metallicity $Z(t)$. This is, however, only a theoretical - not observable - representation for the metallicity distribution in any galactic zone (cf. Ferrini et al. 1992). 

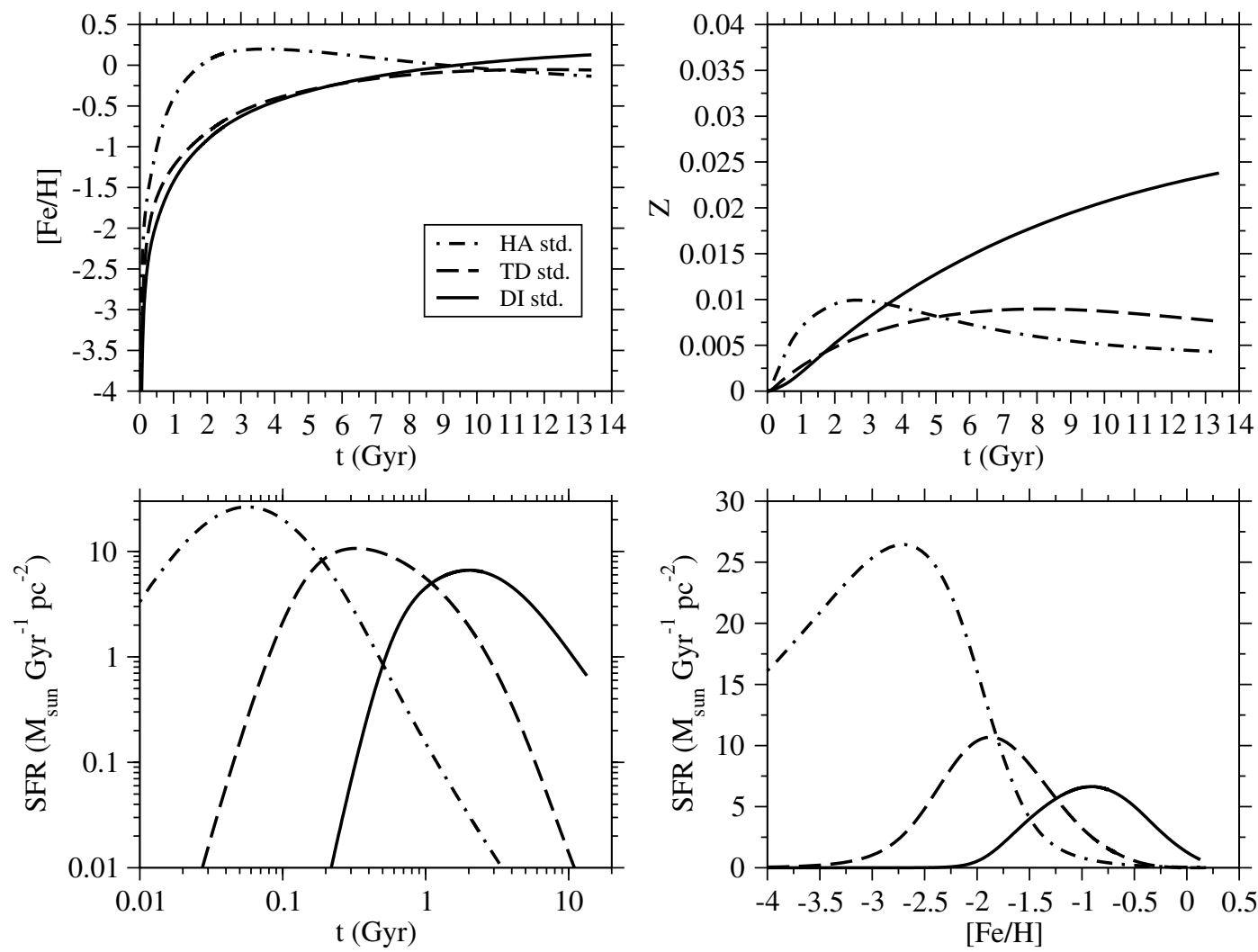

Fig. 1. Standard reference model for our deterministic, constant coefficient, multi-population multizone models. Panels show the evolution of three zones (halo, thick disk, thin disk): clockwise from upper left: metallicity evolutions $([\mathrm{Fe} / \mathrm{H}]$ and $Z$ ), population histogram for stellar metallicity. The SFR for fixed IMF serves as a proxy measure provides a proxy for the number of stars at any instant for high mass stars with comparatively brief main sequence lifetimes.

\section{Stochastic systems with infall}

\subsection{Closed systems}

There is overwhelming evidence that the Galaxy, and most extragalactic stellar systems, are subject to constant forcing by the combined effects of collisions, accretion, and stripping of gas and stars. Our system has accreted at least a few dwarf galaxies in its lifetime - for instance, Sgr and CMa - and the high velocity clouds also seem to be infalling gas that isn't merely recycled from the corona or disk and may originate outside of the Galactic halo (Wakker et al. 1999). The detection of deuterium, $\mathrm{D} / \mathrm{H} \sim 10^{-6}$, in Galactic center molecular clouds is further evidence for an infall rate in the disk of about $1 M_{\odot} \mathrm{yr}^{-1}$ (Lubowich et al. 2000). But before examining the effects of any external mass accretion, we simulated closed systems assuming stochastic halo infall to the thick disk and thick disk infall to the thin disk as a baseline for the open cases.

We modified the standard model by adopting infall rates $\left(f_{\mathrm{H}}, f_{\mathrm{TD}}\right)$ that fluctuate randomly in time with the amplitudes being drawn from a Gaussian distribution with a freely chosen dispersion to mimic shortlived accretion bursts that might occur for infall of halo or thick disk clouds. We maintained a constant timestep for the models, $3 \mathrm{Myr}$, with an infall event every 50 timesteps (150 Myr). In each event, which has impulsive rise and exponential decline, the decay time is specified as a parameter (in these models $100 \mathrm{Myr}$ ). The mass of the total system was kept constant. A variety of models were computed with range of dispersions and amplitudes but these produced the same basic behavior so we will here report only the results for one simulation, shown in Fig. 2. This model assumed that the mean value for infall from each zone was the same as the standard model but with $f_{\mathrm{H}}: N\left(\mu=f_{\mathrm{H}}, \sigma=30 \% f_{\mathrm{H}}\right)$, and $f_{\mathrm{TD}}: N\left(\mu=f_{\mathrm{TD}}, \sigma=30 \% f_{\mathrm{TD}}\right)$ where $N(\mu, \sigma)$ is a normal distribution with mean $\mu$ and dispersion $\sigma$. Compared to the standard model, neither the metallicity distribution nor the star forming history changed significantly for the thin disk for closed system stochastic accretion. The changes seen (Fig. 2) depend on the timing of specific infall events. For instance, enhanced infall early in the evolution of the halo reduces the SFR while a negative fluctuation enhances it; different realizations of a stochastic sample will produce altered histories with the mean behavior mimicking the standard model. Reverse transfer between zones (e.g. blow-out, SNR, etc.) can also be included in this prescription but the results are expected to be similar to those shown in Fig. 2 since the total mass of the system does not change. The model assumptions are similar to those of Copi et al. (1995) and Copi (1997) with one important difference: because we use the full set of model equations, random forcing also causes the star formation rate to vary on its own timescale in response to the accretion fluctuations. The combined effects of dilution and resupply of gas for continued star formation increases the dispersion in each zone's elemental abundance distribution but doesn't alter its mean value. 

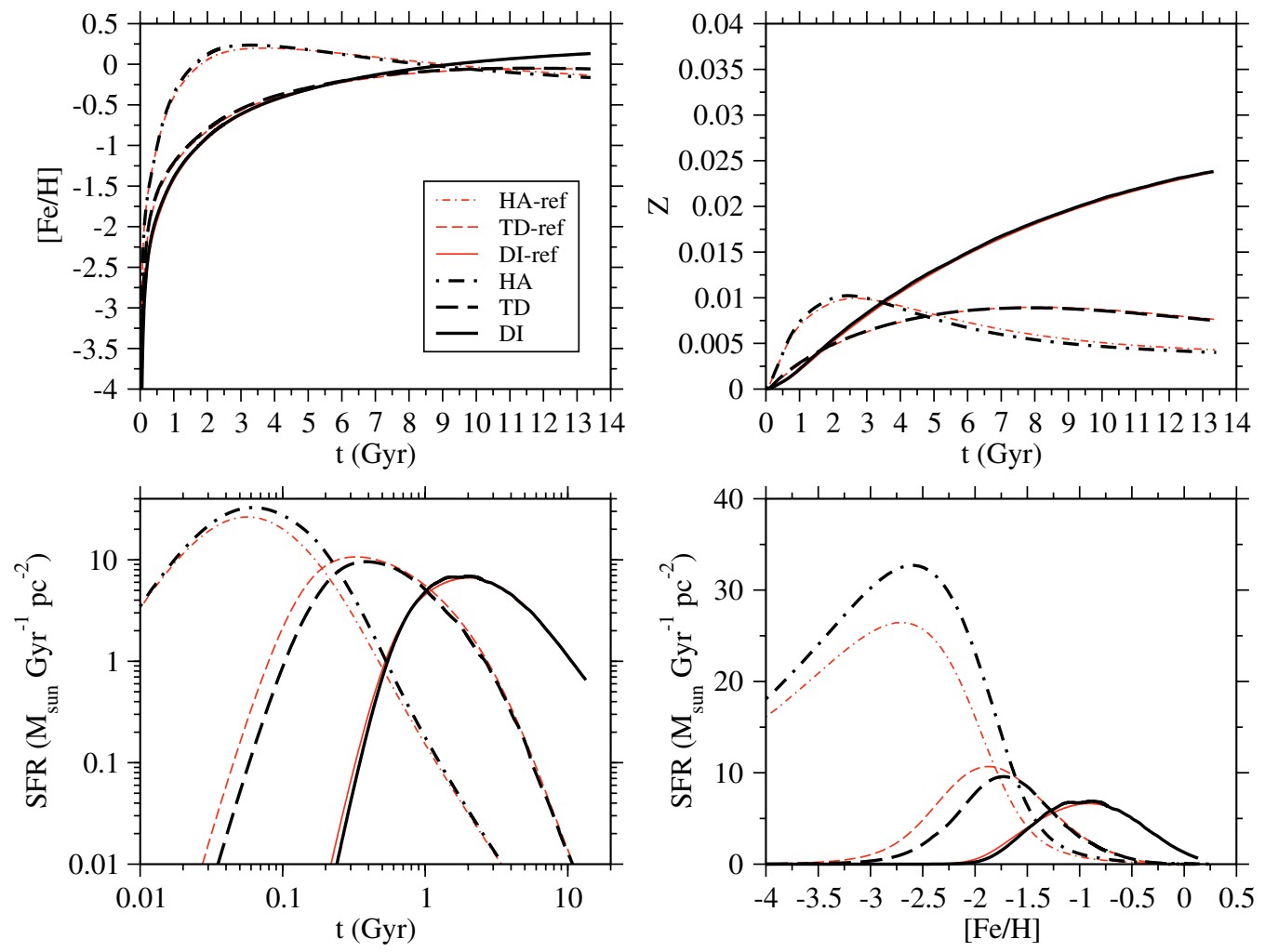

Fig. 2. Stochastic closed system: sample simulation of random infall for a closed system (see text for details). The display is the same as Fig. 1.

\subsection{Open systems}

To study the effects of external gas accretion, perhaps in the form of high velocity clouds or intracluster gas, we performed a variety of simulations for open systems. In all the external diffuse gas, with various assumed metallicities, was added directly to the thin disk. The total mass of the three zones increases by up to $44 \%$ over the simulation. We should emphasize here that our models assume, in effect, a dark matter halo for the bulk of the gravitational potential. We do not need to include the dynamical reaction of the three zones which are passive tracers of the chemical and star forming histories. Thus, although we have models for which $M_{\text {final }} / M_{\text {initial }}$ may be very far from unity, this is still a small change relative to the total galactic mass. Both the amplitudes and intervals between successive events were randomized. The times have been chosen to examine the behavior of the model, not for any particular reason related to the epoch of formation of any particular zone. This contrasts with the parametric study by Chiappini et al. (1997) who by coincidence timed their two infall event model to about the same instants as our simulations, about 1 and $3 \mathrm{Gyr}$, but with the aim of mimicking the growth of the Galactic thin and thick disk. These earlier models also adopted a Schmidt-like power law parametrization for the star formation rate, in contrast to our multiphase approach.

For infall of solar metallicity gas (Fig. 3), the evolution is not drastically changed compared to the standard model after the first few Gyr. The final thin disk metallicity is higher throughout its history, reflecting secondary processing of the already enriched gas. The thin disk metallicity distribution shows a sharp lower cutoff at $[\mathrm{Fe} / \mathrm{H}]=-1.5$ and the metallicity and
SFR stay consistently higher even at the end of the calculation (by about $10 \%$ and a factor of about 4 , respectively) relative to the standard model. For the low metallicity accretion case (Fig. 3), we adopted $0.1 Z_{\odot}$ for gas with the metallicity of the Magellanic Clouds (to mimic gas from the Magellanic Stream). A similar metallicity has been proposed by Wakker et al. (1999) for a high velocity cloud. The final abundances are reduced relative to the standard model but only by about $30 \%$ while the SFR increases by about the same factor as the high- $Z$ case since this depends only on the amount of gas added to the system. But as a consequence, the $[\mathrm{Fe} / \mathrm{H}]$ histogram is essentially unchanged from the standard model although it shows a high end cutoff at $[\mathrm{Fe} / \mathrm{H}] \approx-0.1$ reflecting the dilution by the accreted gas.

Finally, to simulate primordial gas accretion, we show in Fig. 3 the result for stochastic infall for gas with standard Big Bang Nucleosynthesis (SBBN) abundances $\left(X_{0}=0.758\right.$, $\left.Y_{0}=0.242, Z_{0}=0,{ }^{2} \mathrm{D}=6.5 \times 10^{-5}\right)$. The accretion, which again passes directly to the thin disk, increases the system mass by $10 \%$. At this low accretion rate, the thin disk SFR is unchanged. The metallicity evolution curve (top right) illustrates the relatively weak dilution effects that are offset by continuing star formation driven by the infall directly to the thin disk; the final metallicity is reduced by about $20 \%$ relative to the standard model while the histogram for $[\mathrm{Fe} / \mathrm{H}]$ is unchanged. For various infall rates the mass increase for the system is shown in Fig. 4a while the deuterium abundance evolution is shown in Fig. $4 b$.

The early evolution of the ${ }^{2} \mathrm{D}$ abundance is dominated by astration. All models follow the same curve during the period 

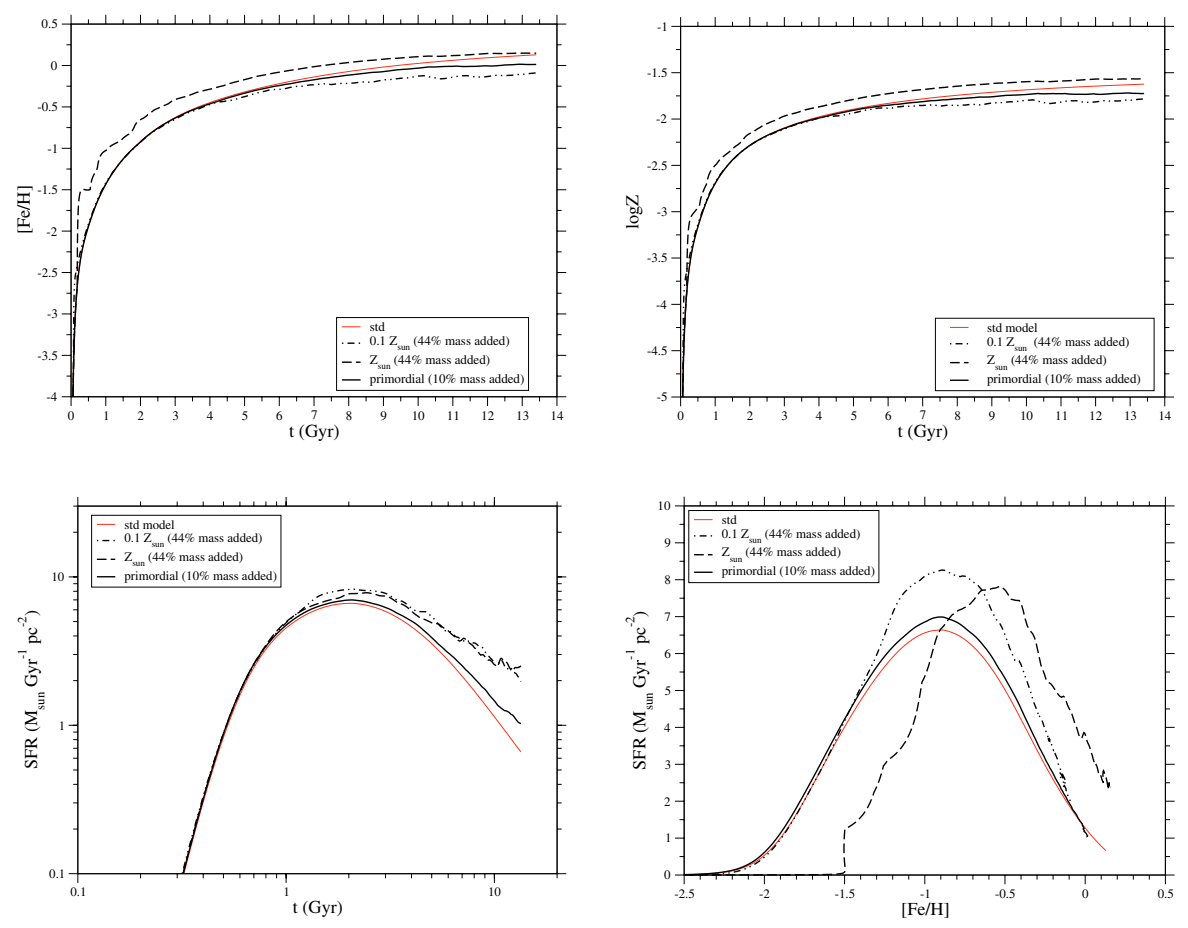

Fig. 3. Open stochastic simulation; in each case, the accretion is directly to the thin disk by an open system and only the thin disk zone results are displayed compared to the standard model. (Dash) Metal-rich $\left(Z=Z_{\odot}\right)$ stochastic accretion (i.e. from the extragalactic environment) and the total mass increases by $44 \%$. Also shown are the cases for (dot-dash) low metallicity (Magellanic Stream simulation) with $44 \%$ added total mass, and (thick continuous) primordial intergalactic gas with $10 \%$ total added mass (see text for details).
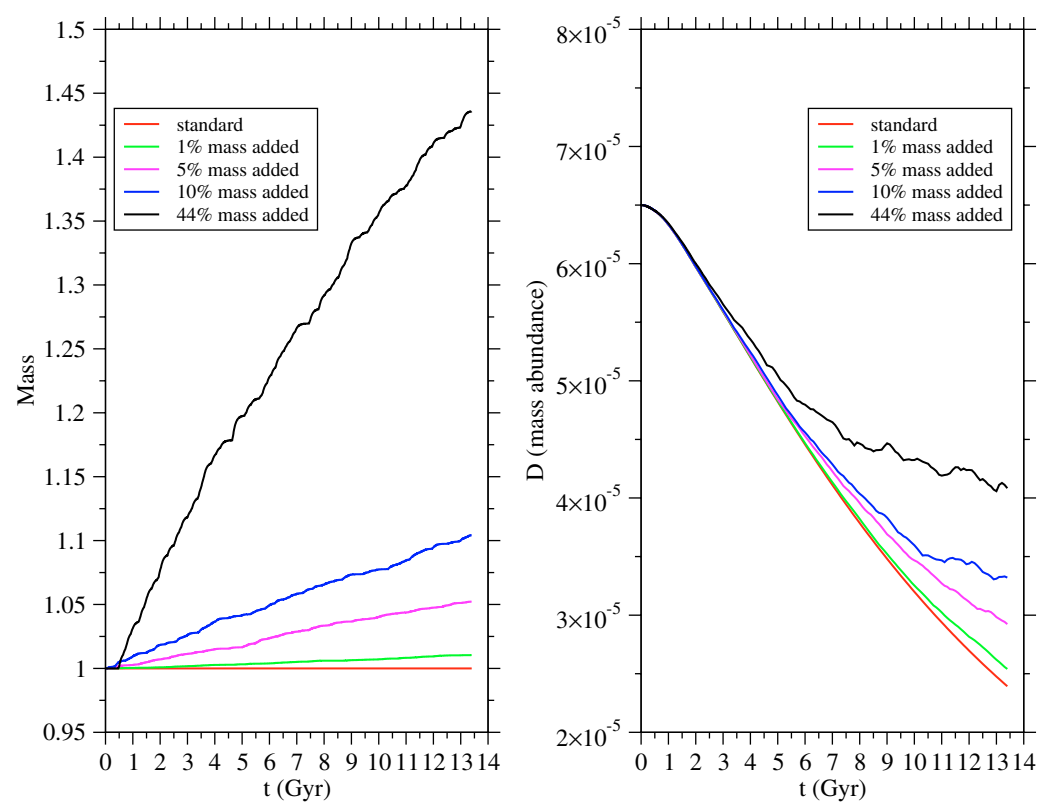

Fig. 4. ${ }^{2} \mathrm{D}$ evolution for the open system described in Fig. 3 in the case of primordial material accretion. The temporal development of total galactic mass a) and ${ }^{2} \mathrm{D}$ thin disk abundance (by mass) b) for a set of open stochastic accretion models with primordial gas accreted. From the top to the bottom the curves refer to: $44 \%, 10 \%, 5 \%, 1 \%$ and no mass added.

of intense halo and thick disk star formation which depletes the internal gas of deuterium. The subsequent evolution for the thin disk is dominated by accretion and the competition between the ${ }^{2} \mathrm{D}$-depleted material shed from the halo and thick disk stars and the external accretion. This reduces the final ${ }^{2} \mathrm{D}$ abundance below SBBN. If the dynamical mixing timescale in the Galaxy is longer than the accretion rate, some residual fluctuations can occur in the interstellar ${ }^{2} \mathrm{D}$ abundance at the current epoch.

Figure 5a shows a sample realization of a single open stochastic model for the primordial case for low mass accretion. Each infall event, of which there are 100 in this model, is assumed to have a short decay time, $\tau=100 \mathrm{Myr}$. In Fig. $5 \mathrm{~b}$ we 

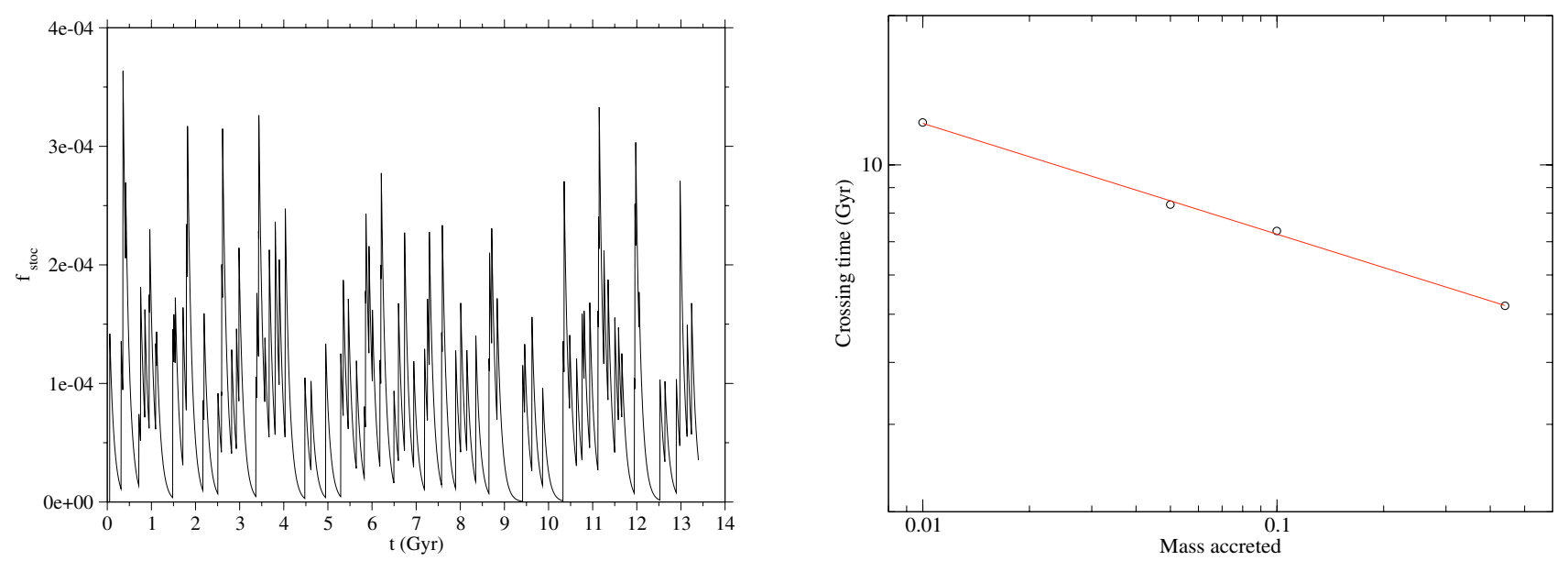

Fig. 5. a) Single realization illustrating the time history for the events for the model shown in Fig. 3 in the case of primordial accretion. b) Dependence of the crossing time for galactic deuterium on fractional accreted mass (see Fig. 4a) for the galaxy for $\Delta^{2} \mathrm{D} /{ }^{2} \mathrm{D}(\mathrm{std})=0.05$. As explained in the text, this is a way to quantify the possible abundance dispersion in a mixed galactic ISM and to illustrate the competition between stellar processing and environmental resupply.

show one way of quantifying the infall models by plotting the time at which the ${ }^{2} \mathrm{D}$ abundance departs by a specified fraction from the predictions of the standard closed model as a function of the fractional accreted mass. Fluctuations in $\mathrm{D} / \mathrm{H}$ of order $5 \%$ are compatible with ISM abundance studies and indicate the influence of infall relative to mixing within the disk. If the disk mixing time is less than the crossing time in Fig. 5b, the process is likely to wash out local fluctuations.

\section{Collisions and stripping}

For any cluster member, collisions are always possible (Mihos 2004). While we cannot model the different types in detail (e.g. major vs. minor mergers, or tidal disruption in the cluster potential), we can study the phase response to a schematic forcing. Collisions were simulated as impulsive events starting at some time $t_{\text {start }}$ and lasting for $\Delta t_{\text {coll }}$ during which time the diffuse gas fraction is set continuously to some reduced value, including complete removal. For the stripping simulations, the clouds were not removed during the events since studies of cluster galaxies imply that only the diffuse gas is removed (e.g. Moore 2004). To test the effect of cloud removal during collisions, we have also performed simulations with various efficiencies of gas removal. During real collisions, galaxies appear to experience episodes of stimulated enhanced star formation. We do not obtain this in our models nor do we include its effect on the metallicity evolution by arbitrary additional assumptions. By keeping the rates that govern star-cloud conversions constant, we ignore (and therefore underestimate) possible triggered bursts, but our approach can accommodate such scenarios when appropriate rates are physically calculated outside the model. Cloud destruction depletes that phase and, for this reason, the SFR drops only after the removal of the diffuse gas on the cloud destruction timescale. At the end of the collision interval, gas is resupplied only internally, i.e. from mass shed by stars that have evolved within the various zones - without additional mass loss from the system - on a timescale determined by stellar evolution and the assumed IMF. This approximates a fast, shortlived analog of stripping that lasts only a short time after which gas removal stops abruptly. In the cases shown here we used durations of 50 and $130 \mathrm{Myr}$ started at two different times: around the peak of the standard model thin disk star formation ( $3 \mathrm{Gyr}$ ), and before the peak in the thin disk standard model but after the peaks of the halo and thick disk (1 Gyr). Different efficiencies were assumed for the fraction of gas removed in a single event (the stripping efficiency) and we used a variety of time profiles. We present only the results for single or double collisions in Figs. 6 and 8. Any plausible combination of waiting times and amplitudes for any of these events produces a superposition of the outcomes of the individual collisions.

To include a dynamically modeled mass loss history within the stripping during a collision, we adopted the numerical results of Quilis et al. (2000), shown for reference in Fig. 7. This stripping history governs all diffuse gas within the galaxy including that returned by stellar mass loss. We find a generic behavior of the systems. The models are quite insensitive to the detailed history of the event but depend strongly on the efficiency of the gas removal in the initial stage of the collision and on the timing of the collision, since the removal of gas throttles the star formation and produces a hiatus in the metallicity production. Since we compute the SFR directly, we see that its sharp drop halts metallicity production for some time, until stellar evolution resupplies disk gas and heavy elements. The recovery is not as complete as first found by Comins \& Shore (1990). We note that in these older models, an improper normalization to the total mass was applied. Consequently the efficiency of replenishment of the gas by stellar death was overestimated. We now find that the decrease in the active mass means the SFR after the collision never reaches its previous levels or those of the standard model and the system remains permanently metal poor. This may reproduce the metallicity histories of dwarf galaxies in clusters that undergo very early tidal interactions while still forming stars (see Figs. 6 and 8).

During a collision, the metallicity initially rises by about $20 \%$ over a very short time, about $\Delta t_{\text {coll }}$. Thereafter, the 

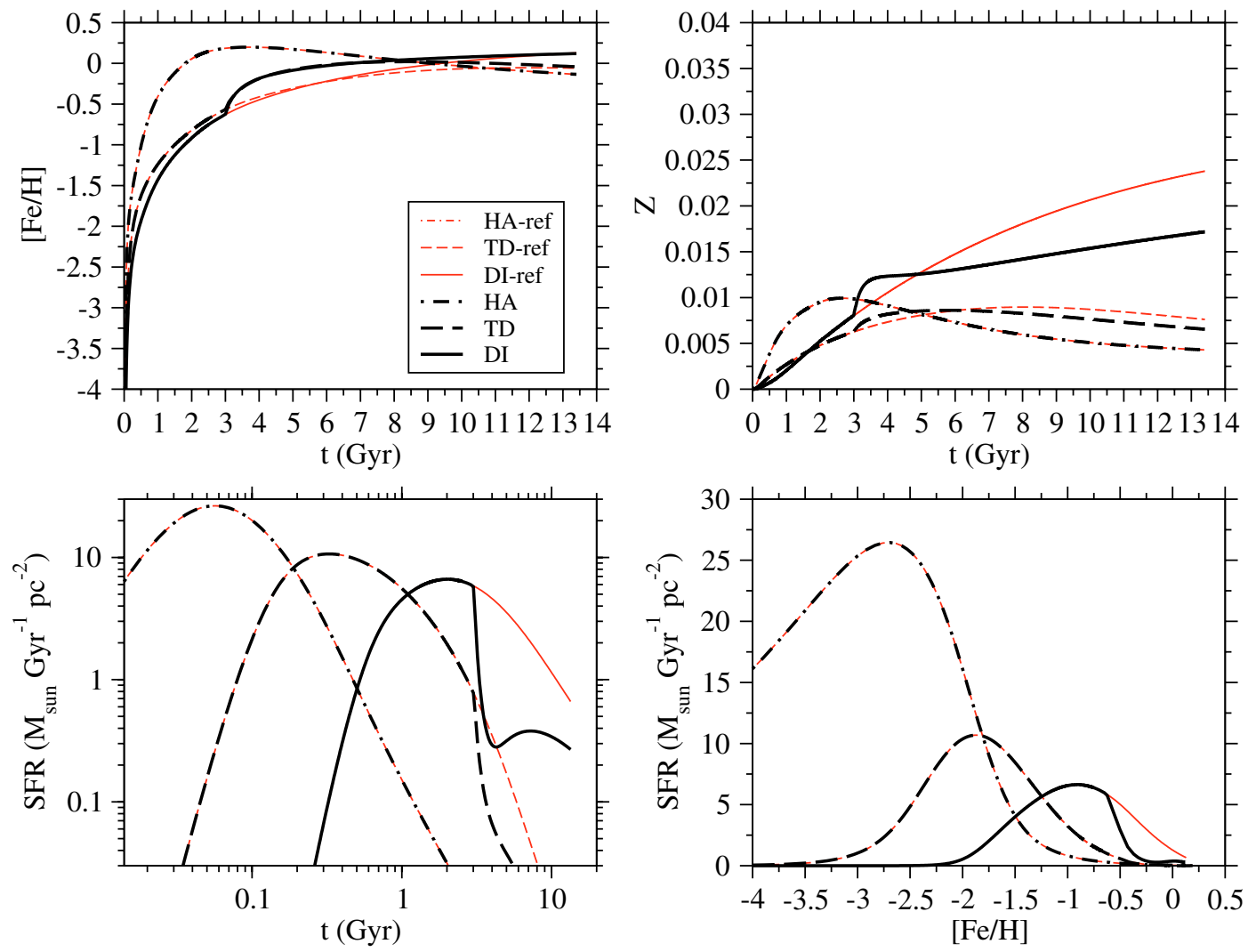

Fig. 6. Collision simulation starting at $t_{\text {start }}=3 \mathrm{Gyr}$ lasting for $\Delta t_{\text {coll }}=50 \mathrm{Myr}$ (see text).

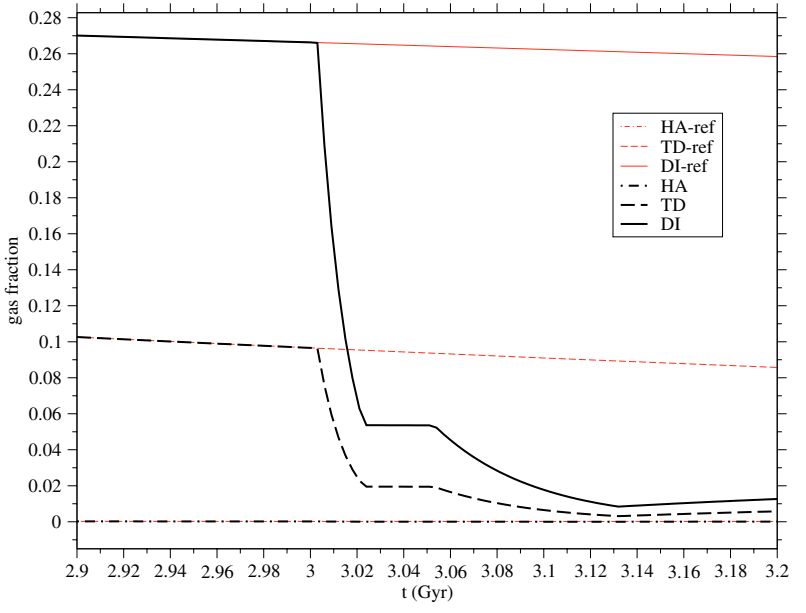

Fig. 7. Time histories for the mass of the galaxy in collision simulations using the mass loss profile from Quilis et al. (2000) for $t_{\text {start }}=3 \mathrm{Gyr}$ and $\Delta t_{\text {coll }}=130 \mathrm{Myr}$.

reduced SFR produces a nearly flat, reduced metal abundance and an upper cutoff to the $[\mathrm{Fe} / \mathrm{H}]$ vs. SFR histogram. To test the sensitivity of the results to the duration and timing of the collision, we varied the two parameters while keeping the Quilis et al. (2000) profile for the mass loss. The results are shown in Fig. 8.

For collisions with $t_{\text {start }}=3 \mathrm{Gyr}$, the upper cutoff is at about $[\mathrm{Fe} / \mathrm{H}]=-0.5$. This is drastically altered for the earlier $t_{\text {start }}$ models for which the thin disk never effectively forms. The histogram is significantly reduced with a peak at around
$[\mathrm{Fe} / \mathrm{H}]=-1.5$ and the thick disk also developed a reduced metallicity, a cutoff is found for $[\mathrm{Fe} / \mathrm{H}] \approx-1$. The final $Z$ for the thin disk is reduced by about $40 \%$ relative to the standard model.

As a final result from the collision simulations, we show in Fig. 9 the deuterium evolution of the models. For this isotope, the curves are almost identical to the infall case for relatively low metallicity gas. This can be understood from the SFR profile. If star formation is suppressed so is astration (e.g. Vidal-Madjar 2002; Lemoine et al. 1999; Hébard et al. 2002). Since we found that the subsequent ${ }^{2} \mathrm{D}$ evolution depends on the infall rate, the final abundance is set mainly by the amount of gas remaining in the system and resupplied from the halo and thick disk. Reducing the star formation for the subsequent history elevates the final abundances relative to the standard model (Lubowich et al. 2000 find this for the Galactic center region, for instance). For multiple collisions, the results for which are shown in Fig. 10b, the ${ }^{2} \mathrm{D}$ is nearly indistinguishable from the standard model.

The effects on the evolution of the light elements is, however, striking since these are produced by the continuing star formation. While in the standard model (Valle et al. 2002) we found the abundance curves to systematically lie above the observations, we now find very different trends depending on the timing and efficiency of the collisions. This difference reflects the changes in the SFR and subsequent supernova activity and the change in the cosmic ray spallation production in the altered diffuse gas. The histories depend sensitively on the timing of the collisions, far more than for the heavy elements, and argue 

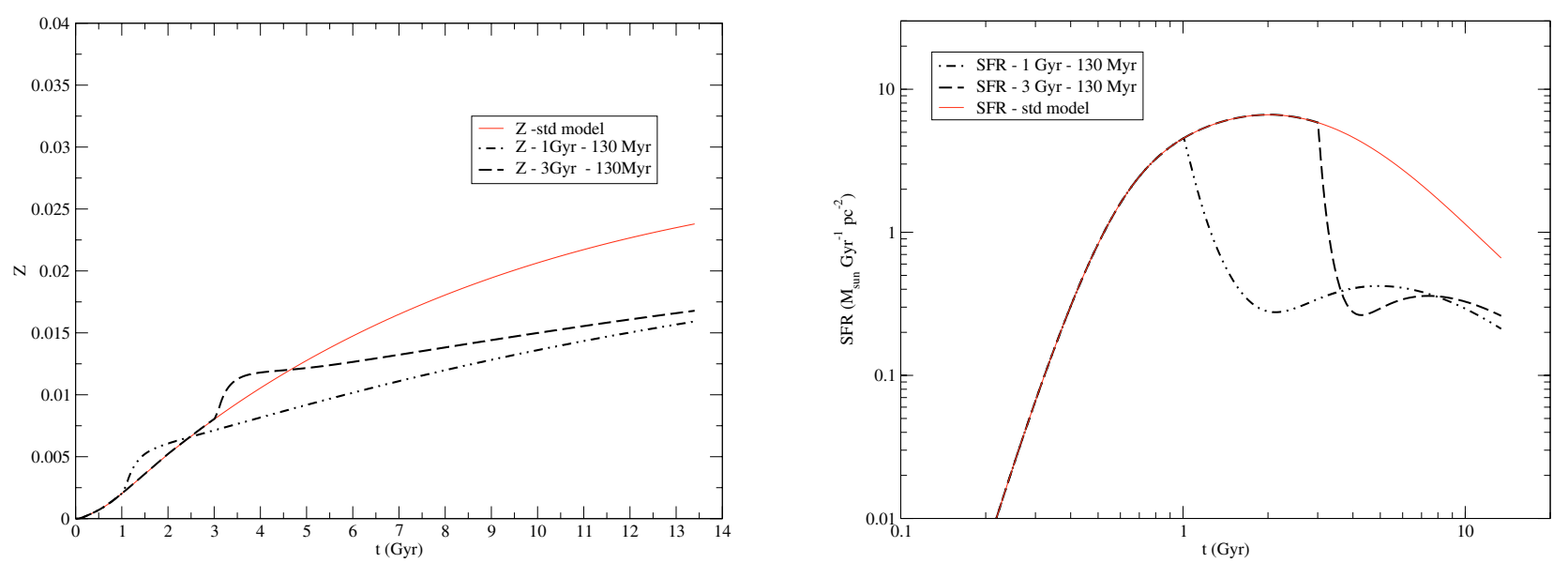

Fig. 8. Collision simulations with $t_{\text {start }}=1 \mathrm{Gyr}, t_{\text {start }}=3 \mathrm{Gyr}$ and $\Delta t_{\text {coll }}=130 \mathrm{Myr}$. Residual gas $3 \%$ after $\Delta t_{\text {coll }}$. Mass loss profiles were as in Quilis et al. (2000). Note: In this and all subsequent figures we display only the thin disk evolution.
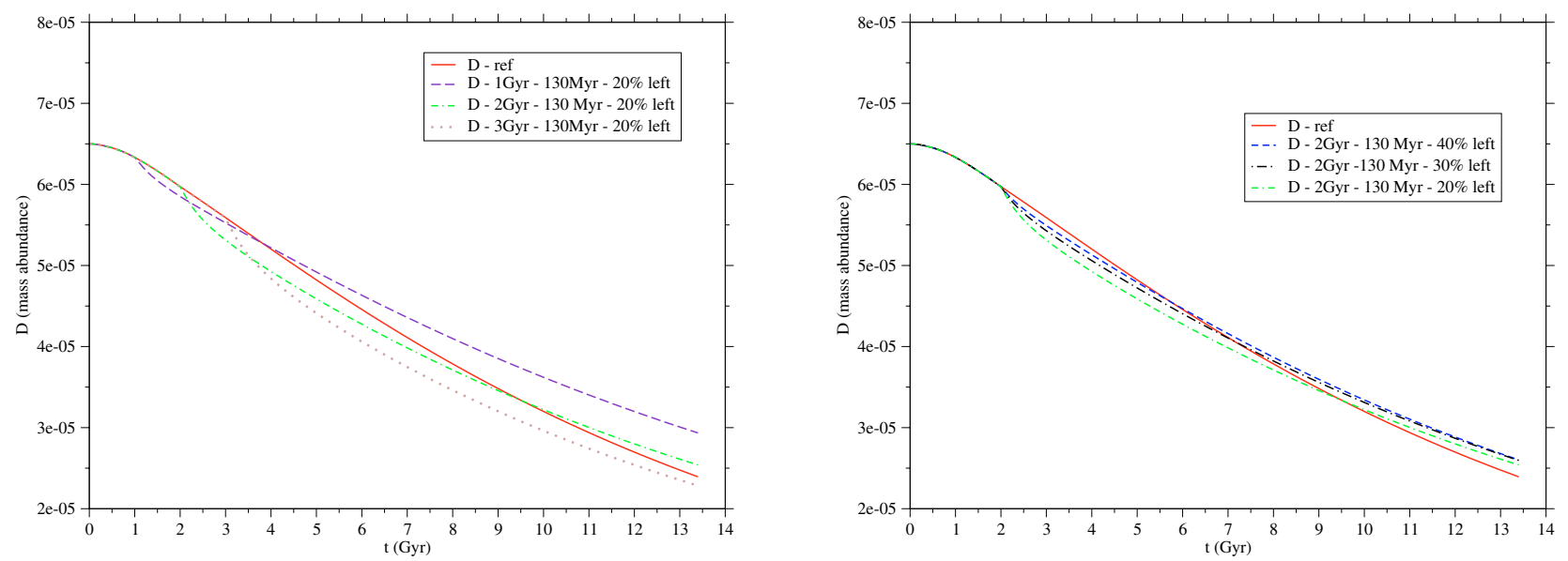

Fig. 9. a) Thin disk D abundances in collision depending on starting time; b) D depending on fraction of gas removed.

against using these species to reconstruct a unique galactic history of star formation. We show a sample of single and multiple collision results in Fig. 10. In each simulation, we removed $\approx 70 \%$ of the diffuse gas present in the system at the onset of the collision event, so these likely represent an upper limit for the reduction of the final abundances. These simulations suggest that after a few strong collisions (and here we don't include the distinction between a major and minor merging event since the models are not dynamical) the final abundances can be altered from the standard model by as much as a factor of three.

\subsection{Stripping simulations}

In our treatment, stripping by an external medium and impulsive collision events are similar in their effects. We have implemented the effects of ram pressure from an intracluster medium by adopting a characteristic timescale of $1.3 \mathrm{Gyr}$ for the event and varying only the initial time for the process using the Quilis et al. (2000) mass loss profile although their timescale is an order of magnitude shorter. Once again, because of the decline of the SFR, the timing of the event is important. If the stripping begins early in the history of the galaxy the thin disk is stunted in its growth and the metallicity is significantly reduced compared to the standard model. The histories adopted here are for single infall episodes (in the sense of the galaxy falling in the cluster potential) and contrast with those used for simulations of ram pressure stripping of Virgo cluster galaxies by Vollmer et al. (2001) who find a ram pressure history that is approximately Lorentzian in time with a $F W H M$ of about 200 Myr (see also Vollmer et al. 2004, and references therein). Again, our prescription is general, not specifically dynamical, and can be adapted to any stripping history when combined with a model for the galaxy motion within a realistic cluster potential and background.

We show our results for two simulations, one starting after the peak of the halo formation but before the thin disk starts to form and the other well after the start of star formation in the thin disk. The final SFR is almost a factor of 10 lower than the standard model and the metallicity is reduced by about $40 \%$. The overall appearance of the system is similar to the collision case, the longer timescale for the continued gas removal having little additional effect compared to the impulsive loss since the SFR is so drastically reduced and the thin disk is severely suppressed (Fig. 11). The models yield behavior that resembles the results of de Mello et al. (2002) who find that "... repeated encounters, experienced by galaxies in dense environments, 

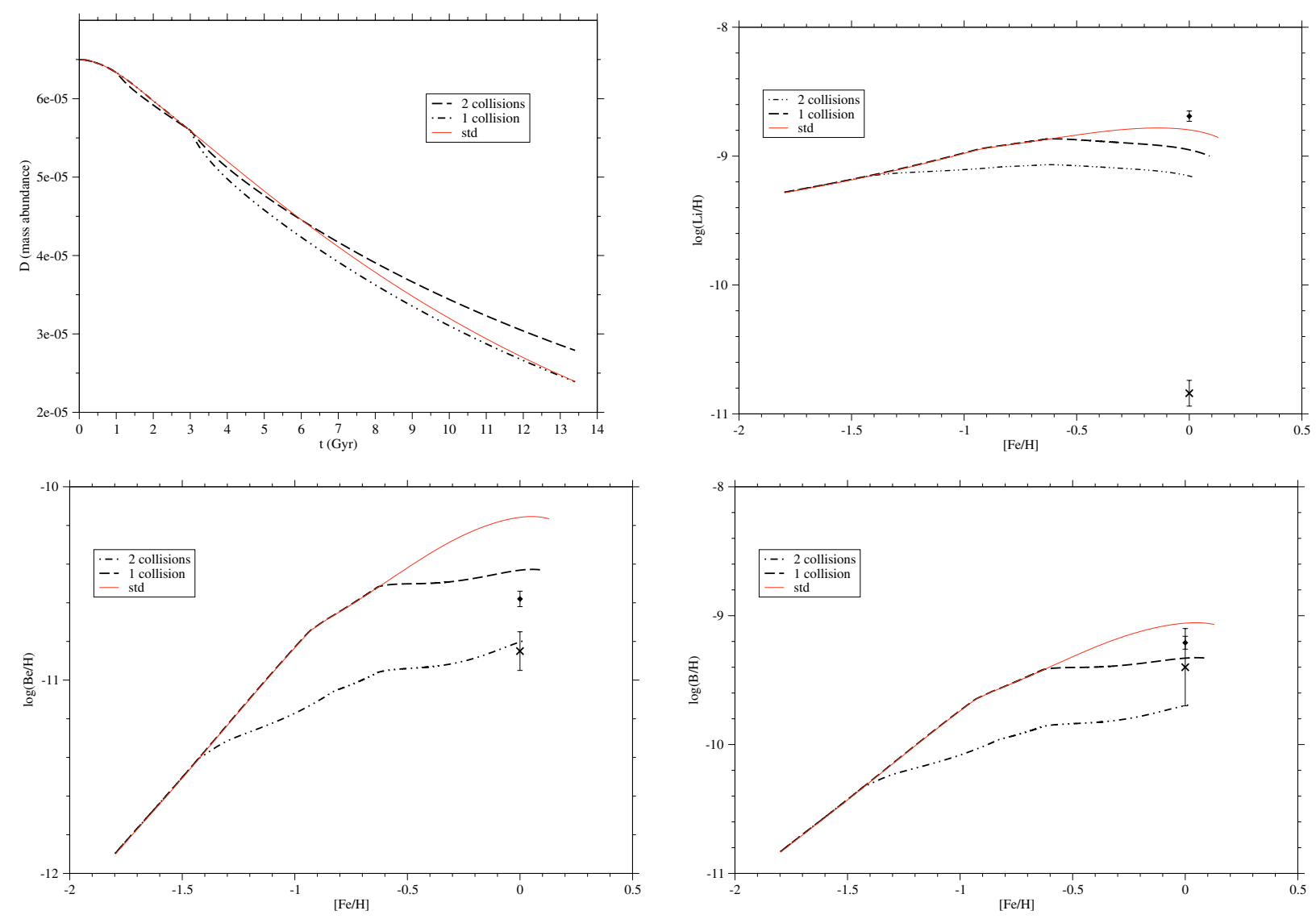

Fig. 10. Comparative evolution of the light elements for two collisional models for the thin disk zone (see text): a single collision as in Fig. 8 with $t_{\text {start }}=3 \mathrm{Gyr}$ and $\Delta t_{\text {coll }}=130 \mathrm{Myr}$ (dashed line) and the case of two collisions with $t_{\text {start }}=1$ and 3 Gyr but the same duration (dot-dashed line). The panels show, in order from the top, ${ }^{2} \mathrm{D}$, total $\mathrm{Li},{ }^{9} \mathrm{Be}$, and total $\mathrm{B}$. For $\mathrm{Li},{ }^{9} \mathrm{Be}, \mathrm{B}$, the meteoritic and the solar photospheric abundances (from Grevesse \& Sauval 1998) are indicated by a diamond and a cross, respectively. In all cases, the diffuse gas remaining after each collision was $30 \%$ of its initial value.
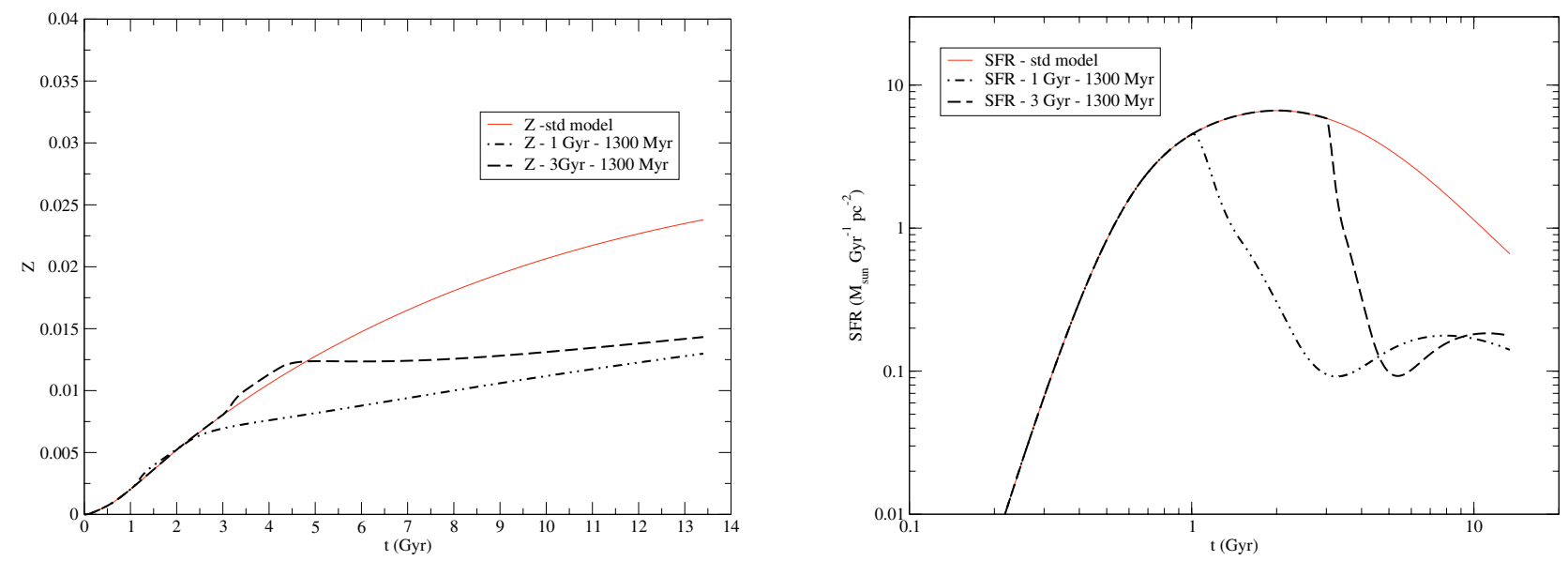

Fig. 11. Stripping simulations comparison with $t_{\text {start }}=1$ and $t_{\text {start }}=3 \mathrm{Gyr}, \Delta t_{\text {coll }}=1.3 \mathrm{Gyr}$ using the Quilis et al. (2000) mass loss profile. The residual gas fraction was $3 \%$ after $\Delta t_{\text {coll }}$.

removes gas from the galaxies as well as leads to an inhibition of the formation of the molecular gas from the atomic phase. The similarities in star-formation efficiency of the dense environments and field galaxies suggest that the physical processes controlling the formation of stars from the molecular gas are local rather than global".
For the observable HR diagram, we expect a range of morphologies. For systems undergoing strong stripping, the giant branch should be diffuse with the bulk of the older stars being formed with lower metallicity and the higher metallicity stars showing an almost continuous distribution in color at any luminosity. We will report the detailed simulations in a future paper 

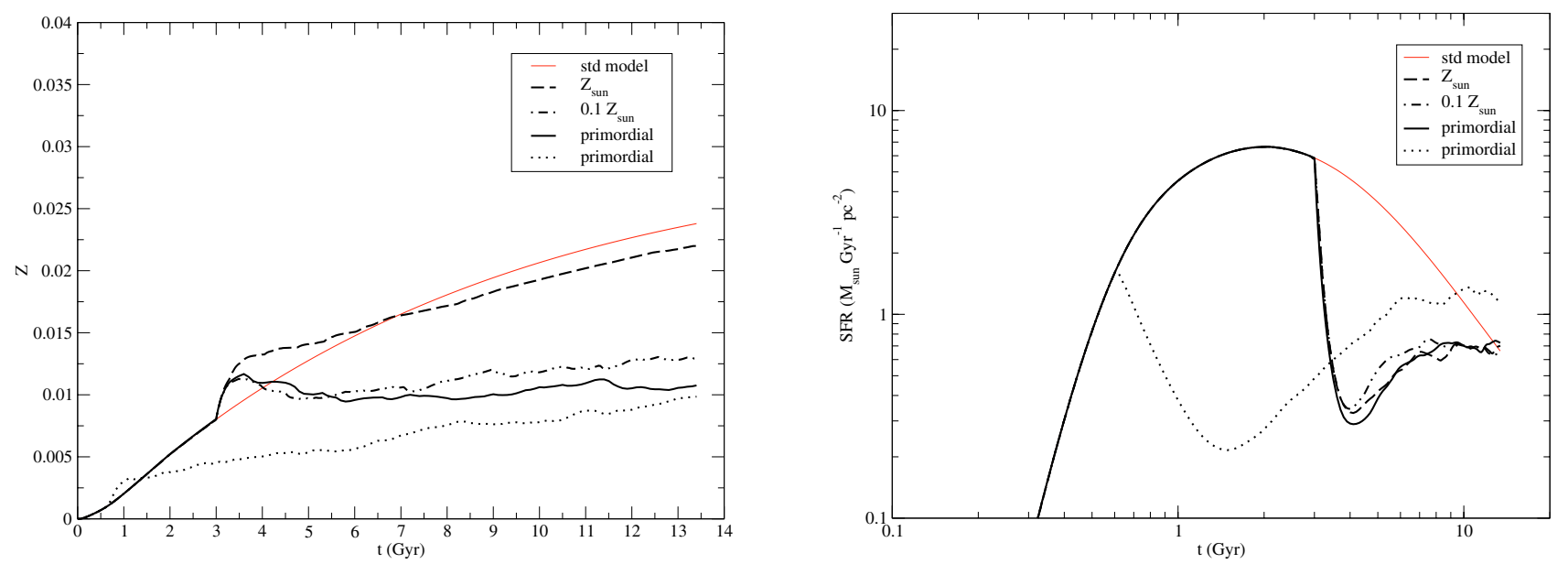

Fig. 12. Combined effects of collision and environmental infall. The collision event was assumed to have $t_{\text {start }}=3 \mathrm{Gyr}$ and $\Delta t_{\text {coll }}=50 \mathrm{Myr}$ (see text for details). After the collision, the models assume stochastic infall, in the same way as in Fig. 3, but the re-accretion after the end of the collision. About $40 \%$ of the total initial mass was removed during the collision and then about $10 \%$ was restored during the subsequent re-accretion. Three principal cases of environmental infall are considered: low metallicity gas $\left(0.1 Z_{\odot}\right)$ (dot-dash), metal rich ambient gas $\left(Z_{\odot}\right)$ (dash), and primordial material (black continuous). One additional case is shown for re-accretion of primordial material (dotted line), starting the collision at an earlier epoch, $0.6 \mathrm{Gyr}$; for this last case, $25 \%$ of the total initial mass is subsequently re-accreted.

but point out that in any galactic model the output from any of the simulations reported here can be inserted to predict colormagnitude diagrams as a function of time (e.g. Cignoni et al. 2003).

\section{Fully open systems with combined stripping and re-accretion}

As a final simulation, we assume that both collisional stripping and accretion of ambient gas can occur (and, as Vollmer et al. 2001 point out, also re-accretion of stripped gas). The results are shown in Fig. 12 for relatively low metallicity accretion $\left(0.1 Z_{\odot}\right)$, metal rich ambient gas $\left(Z_{\odot}\right)$ and primordial material. Both the cases $0.1 Z_{\odot}$ and $Z_{\odot}$ enriched relative to primordial abundances that provide an appropriate range consistent with observations of clusters. The dominant effect comes from the removal of gas unless the infall rate is extremely high. As we have shown in the previous sections, accretion doesn't simply dilute the abundances (Casuso \& Beckman 2004). That may be true in a closed box-type approach or one with a formally assumed SFR. We see instead that - depending on the metallicity of the accreted material and the timing and rates of the stripping and filling events - the new gas powers further star formation and the resulting abundances are never reduced by more than about $40 \%$. The most divergent case is for high metallicity accretion where the effects of the collision are, as expected, completely erased although the reduced star formation over most of the system's history means the high metal stars are comparatively rare relative to the standard model.

We qualitatively anticipate one more interesting outcome of these combined "stripping and filling" scenarios. Such postcollision systems may display anomalous color-magnitude diagrams with double red giant branches and strange agemetallicity relations. For instance, the model in Fig. 12 should show a metal rich population that is older than a rarer metal poor disk population. This is just reversed from the standard model and collisional stripping-only models. This can be understood by weighting $Z(t)$ by the time dependent SFR; although not a large effect in the models we computed, the fact that the $Z$ curve is not monotonic produces the gap in age and the lower metallicity for the more recently formed stars. The greater the rate of resupply, the greater the contrast between the two populations. Perhaps such color-magnitude exist for dwarf systems? It would be interesting to test the picture for cases where the stripping is more devastating and the infall just now restarting. This "burst in reverse" behavior would likely be enhanced in systems that experience stimulated bursts of star formation during the stripping event.

\section{Summary}

Our aim here has been to display the behavior of a galactic model to a wide range of physical processes that are expected to be important during some stage of Galactic history. Because these calculations are restricted to modeling a local region of the disk with multiple vertical stratification - but without dynamics - extended to a schematicized model for the whole galaxy, a detailed comparison with the evolution of the Solar neighborhood and the Galactic disk is beyond our capabilities. But we have found a consistent behavior in the presence of accretion and stripping that resemble that seen in both our and other galaxies. From these simulations we conclude that it is unlikely that one can uniquely reconstruct the star formation history of the Galaxy solely from the metallicity distribution, especially for the light elements. Finally, we note that it is now computationally possible to merge our approach with $N$-body SPH modeling to follow the gas and population evolution selfconsistently during collisions and also for clusters. An important feature of the multiphase models, the separation of the gas into distinct diffusive and cloud phases, can also be extended to include enhanced stimulated star formation during collisions. This hybrid modeling would be able to follow the SFR and 
metallicity evolution explicitly in each individual galaxy within cosmological simulations.

Acknowledgements. We thank Michele Cignoni, Federico Ferrini, Pepe Franco, Cathy Horellou, Joachim Köppen, Francesco Palla, Jan Palouš, Pier Prada Moroni, and Nikos Prantzos for discussions. We also thank the referee, Jesper Sommer-Larsen, for valuable advice and criticisms. Giada Valle wish to thank Matteo Dell'Omodarme for remarkably patient and invaluable help and comments. This work was supported, in part, by COFIN2000 from MIUR.

\section{References}

Casuso, E., \& Beckman, J. E. 2004, A\&A, 419, 181

Chiappini, C., Matteucci, F., \& Gratton, R. 1997, ApJ, 477, 765

Cignoni, M., Prada Moroni, P. G., \& Degl'Innocenti, S. 2003, Mem. SAIt, 74, 899

Comins, N. F., \& Shore, S. N. 1990, A\&A, 237, 345

Copi, C. J., Schramm, D. N., \& Turner, M. S. 1995, ApJ, 455, L95

Copi, C. J. 1997, ApJ, 487, 704

de Mello, D. F., Wikland, T., \& Maia, M. A. G. 2002, A\&A, 381, 771

Ferrini, F. 1991, in Chemical and Dynamical Evolution of Galaxies, ed. F. Ferrini, F. Matteucci, \& J. Franco (Pisa: ETS), 511

Ferrini, F., Matteucci, F., Pardi, M. C., \& Penco, U. 1992, ApJ, 387, 138

Ferrini, F., Mollà, M., Pardi, M. C., \& Diaz, A. 1994, ApJ, 427, 745

Franco, J., \& Shore, S. N. 1984, ApJ, 285, 813

Galli, D., Palla, F., Ferrini, F., \& Penco, U. 1995, ApJ, 443, 536

Gerola, H., \& Seiden, P. E. 1978, ApJ, 223, 129

Grevesse, N., \& Sauval, A. J. 1998, SSR, 85, 161

Hébard, G., Friedman, S. D., Krok, J. W., et al. 2002, Planet. Space Sci., 50, 1169

Hellsten, U., \& Sommer-Larsen, J. 1992, MNRAS, 255, 650

Hensler, G. 2003, in CNO in the Universe, ed. C. Charbonnel, D. Schaerer, \& G. Meynet (San Francisco: ASP), ASP Conf. Ser., 304,371

Kroupa, P. 2002, Science, 295(5552), 82

Lemoine, M., Audouze, J., Ben Jaffel, L, Feldman, P., et al. 1999, New Astr., 4, 231
Lynden-Bell, D. 1975, Vistas Astr., 19, 299

Lubowich, D. A., Pasachoff, J. M., Balonek, T. J., et al. 2000, Nature, 405,1025

Matteucci, F. 2001, The Chemical Evolution of the Galaxy (Dordrecht: Kluwer)

Matteucci, F., \& Chiosi, C. 1983, A\&A, 123, 121

Mihos, J. C. 2004, in Clusters of Galaxies: Probes of Cosmological Structure and Galaxy Evolution, ed. J. S. Mulchaey, A. Dressler, \& A. Oelmer (Cambridge: Cambridge Univ. Press), in press

Moore, B. 2004, in Clusters of Galaxies: Probes of Cosmological Structure and Galaxy Evolution, ed. J. S. Mulchaey, A. Dressler, \& A. Oelmer (Cambridge: Cambridge Univ. Press), in press

Pagel, B. E. J. Nucleosynthesis and Chemical Evolution of Galaxies (Cambridge: Cambridge Univ. Press)

Pardi, M. C., \& Ferrini, F. 1994, ApJ, 421, 491

Perdang, J. Lejeune, A. 1996, A\&AS, 119, 231

Quilis, V., Moore, B., \& Bower, R. 2000, Science, 288, 1617

Schulman, L. S., \& Seiden, P. E. 1986, Science, 233, 425

Shore, S. N., \& Ferrini, F. 1995, Fund. Cosm. Phys., 16, 1

Shore, S. N., \& Franco, J. 2003, in Cosmic Evolution and Galaxy Formation: Structure, Interactions, and Feedback, The 3rd Guillermo Haro Astrophysics Conference, ed. by J. Franco, L. Terlevich, O. Lòpez-Cruz, and I. Aretxaga. (ASP), ASP Conf. Proc., 215, 79

Shore, S. N., \& Galli, D., 2003, Ap\&SS, 284, 809S

Tinsley, B. M. 1980, Fund. Cosm. Phys., 5, 287

Travaglio, C., Galli, D., Gallino, R., et al. 1999, ApJ, 521, 691

Valle, G., Ferrini, F., Galli, D., \& Shore, S. N. 2002, ApJ, 566, 252

Van Gorkem, J. H. 2004, in Clusters of Galaxies: Probes of Cosmological Structure and Galaxy Evolution, ed. J. S. Mulchaey, A. Dressler, \& A. Oelmer (Cambridge: Cambridge Univ. Press), in press

Vidal-Madjar, A. 2002, Planet. Space Sci., 50, 1161

Vollmer, B., Cayatte, V., Balcowski, C., \& Duschl, W. J. 2001, AJ, 561, 708

Vollmer, B., Balkowski, C., Cayatte, V., van Driel, W., \& Huchtmeier, W. 2004, A\&A, 419, 35

Wakker, B. P., Howk, j. C., Savage, B. D., van Woerden, H, et al. 1999, Nature, 402, 388 